\title{
AIE Regimen
}

National Cancer Institute

\section{Source}

National Cancer Institute. AIE Regimen. NCI Thesaurus. Code C67484.

A regimen consisting of cytarabine (Ara-C), idarubicin and etoposide, used as an induction treatment for pediatric acute myeloid leukemia (AML). 